\title{
Erratum: Impacts of non-geometric moduli on effective theory of 5D supergravity
}

\author{
Yutaka Sakamura ${ }^{a, b}$ and Yusuke Yamada ${ }^{c}$ \\ ${ }^{a}$ KEK Theory Center, Institute of Particle and Nuclear Studies, KEK, \\ Tsukuba, Ibaraki 305-0801, Japan \\ ${ }^{b}$ Department of Particles and Nuclear Physics, \\ The Graduate University for Advanced Studies (Sokendai), \\ Tsukuba, Ibaraki 305-0801, Japan \\ ${ }^{c}$ Department of Physics, Waseda University, \\ Tokyo 169-8555, Japan \\ E-mail: sakamura@post.kek.jp, yuusuke-yamada@asagi.waseda.jp
}

ERRATUM TO: JHEP11(2013)090

ABSTRACT: We correct the relation between the physical size of the extra dimension and the VEV of the moduli, and the expressions that follow from it. Errors of the sub-leading terms in section 4 are also corrected.

\section{ARXIV EPRINT: 1307.5585}

1. The two sentences from the seventh line below eq. (3.2) should be corrected as "In our derivation of (3.1), it follows that $\left\langle\phi_{C}\right\rangle=\left\langle\Phi_{C}^{2 / 3}\right\rangle[16]$. Therefore, we find that $L_{\text {phys }}=\left(-\left\langle\Omega_{\mathrm{eff}}\right\rangle / 3\right)^{3 / 2} / M_{\mathrm{Pl}}$."

2. In the first item below eq. (3.6), the relation $\operatorname{Re}\left\langle T_{\text {rad }}\right\rangle=\left\langle\hat{\mathcal{N}}^{1 / 3}\right\rangle$ should be replaced with $L_{\text {phys }}=\left\langle\hat{\mathcal{N}}^{1 / 2}\right\rangle=\left(\operatorname{Re}\left\langle T_{\text {rad }}\right\rangle\right)^{3 / 2}$, and the KK mass $m_{\mathrm{KK}}=\pi /\left\langle\hat{\mathcal{N}}^{1 / 3}\right\rangle$ should be corrected as $m_{\mathrm{KK}}=\pi /\left\langle\hat{\mathcal{N}}^{1 / 2}\right\rangle$.

3. In eqs. (4.1) and (4.13), the argument of $\mathcal{Z}$ should $\operatorname{read} d_{a} \cdot \operatorname{Re} T$.

4. Eq. (4.12) should read

$$
\Omega_{\mathrm{eff}}=-3 \hat{\mathcal{N}}^{1 / 3}+\frac{\left(n_{V}-\bar{n}_{H}+1\right) \zeta(3)}{32 \pi^{2} \hat{\mathcal{N}}^{2 / 3}}+\cdots,
$$

5. The second line below eq. (4.13), $L_{\text {phys }}=\left\langle\hat{\mathcal{N}}^{1 / 3}\right\rangle \gg 1$ should read $L_{\text {phys }}=\left\langle\hat{\mathcal{N}}^{1 / 2}\right\rangle \gg 1$. 
6. Eq. (4.17) should read

$$
\xi_{I_{\mathrm{o}}} \equiv \frac{\partial \xi}{\partial T^{I_{\mathrm{o}}}}=\sum_{a} \frac{n_{a} d_{a I_{\mathrm{o}}}\left(d_{a} \cdot \operatorname{Re} T\right)}{16 \pi^{2}} \ln \left(1-e^{-2\left|d_{a} \cdot \operatorname{Re} T\right|}\right) .
$$

7. Eq. (4.20) should read

$$
\begin{aligned}
V_{\mathrm{pot}}= & e^{K}\left(D_{I} W K^{I \bar{J}} D_{\bar{J}} \bar{W}-3|W|^{2}\right) \\
= & \frac{1}{\hat{\mathcal{N}}}\left\{\frac{2 \hat{\mathcal{N}}(a A)^{2} e^{-2 a \tau_{s}}}{3 C_{s} \tau_{s}\left(1-\frac{\delta}{6}\right)}+4 a \tau_{s}\left(1+\frac{\epsilon}{3}+\frac{\delta}{6}\right) W_{0} A e^{-a \tau_{s}} \cos \left(a \rho_{s}\right)\right\} \\
& +\frac{6 \xi W_{0}^{2}}{\hat{\mathcal{N}}^{2}}\left\{1-\frac{2 C_{s} \tau_{s}^{3}}{3 \xi}\left(\epsilon-\frac{\delta}{4}\right)\right\}+\cdots,
\end{aligned}
$$

8. eq. (4.22) should read

$$
\begin{aligned}
\left\langle\tau_{b}^{3}\right\rangle & \simeq\langle\hat{\mathcal{N}}\rangle=\frac{3 \xi W_{0} e^{a\left\langle\tau_{s}\right\rangle}}{a\left\langle\tau_{s}\right\rangle A}\left\{1-\frac{\epsilon}{3}\left(1+\frac{2 C_{s} \tau_{s}^{3}}{\xi}\right)-\frac{\delta}{6}\left(1-\frac{C_{s} \tau_{s}^{3}}{\xi}\right)\right\}, \\
\left\langle\tau_{s}\right\rangle & \simeq\left(\frac{\xi}{C_{s}}\right)^{1 / 3}\left\{1-\frac{2 \epsilon}{3}\left(1+\frac{C_{s} \tau_{s}^{3}}{3 \xi}\right)-\frac{\delta}{6}\left(1-\frac{C_{s} \tau_{s}^{3}}{3 \xi}\right)\right\} \equiv \tau_{s}^{(0)}, \\
\cos \left\langle\rho_{s}\right\rangle & =-\operatorname{sign}\left(W_{0} A\right),
\end{aligned}
$$

9. In the fifth line below eq. (4.23), $L_{\text {phys }}=\left\langle\hat{\mathcal{N}}^{1 / 3}\right\rangle$ should be replaced with $L_{\text {phys }}=\left\langle\hat{\mathcal{N}}^{1 / 2}\right\rangle$.

10. Eq. (4.24) should read

$$
\begin{aligned}
& m_{\tau_{b}} \simeq\left\langle\frac{12 \sqrt{6 \xi} W_{0}}{\hat{\mathcal{N}} \sqrt{a \tau_{s}}}\left\{1+\frac{C_{s} \tau_{s}^{3}}{9 \xi}\left(\epsilon+\frac{11}{4} \delta-\zeta\right)+\frac{1}{9}(-8 \epsilon+3 \delta+\zeta)\right\}\right\rangle, \\
& m_{\rho_{b}} \simeq 0, \quad m_{\tau_{s}}, m_{\rho_{s}} \simeq\left\langle\frac{4 a \tau_{s} W_{0}}{\sqrt{\hat{\mathcal{N}}}}\left\{1+\frac{\epsilon}{3}+\frac{\delta}{12}\right\}\right\rangle,
\end{aligned}
$$

where $\zeta \equiv \xi^{\prime \prime \prime} / C_{s}$.

11. Eq. (4.25) should be replaced with

$$
V_{\min } \simeq-\left\langle\frac{18 \xi W_{0}^{2}}{\left(2 a \tau_{s}+1\right) \hat{\mathcal{N}}^{2}}\right\rangle .
$$

12. Eq. (4.28) should read

$$
\left\langle\tau_{s}\right\rangle=\tau_{s}^{(0)}+\frac{3}{2 a^{2} \tau_{s}^{(0)}}\left\{1-\frac{\epsilon}{9}\left(2+\frac{5 C_{s} \tau_{s}^{(0) 3}}{\xi}\right)+\frac{\delta}{9}\left(1+\frac{C_{s} \tau_{s}^{(0) 3}}{\xi}\right)\right\}
$$


13. Eq. (4.29) should read

$$
\left\langle\frac{F^{T_{s}}}{T_{s}+\bar{T}_{s}}\right\rangle \simeq-\left\langle\frac{K^{T_{s} \bar{T}_{s}} W_{\bar{T}_{s}}+K^{T_{s} \bar{I}} K_{\bar{I}} \bar{W}}{2 \tau_{s} \sqrt{\hat{\mathcal{N}}}}\right\rangle \simeq \frac{m_{3 / 2}}{a \tau_{s}^{(0)}} \ll\left\langle\frac{F^{T_{b}}}{T_{b}+\bar{T}_{b}}\right\rangle .
$$

14. Eq. (4.30) should read

$$
\left\langle\frac{F^{\phi_{C}}}{\phi_{C}}\right\rangle=\frac{1}{3}\left\langle K_{I} F^{I}\right\rangle+m_{3 / 2}=\mathcal{O}\left(\frac{m_{3 / 2}}{\hat{\mathcal{N}}^{4 / 3}}\right) \ll\left\langle\frac{F^{T_{s}}}{T_{s}+\bar{T}_{s}}\right\rangle,
$$

15. Eq. (4.31) should read

$$
M_{r}=\left\langle F^{I} \partial_{I} \ln \left(\operatorname{Re} f_{\mathrm{eff}}^{r}\right)\right\rangle \simeq\left\langle\frac{F^{T_{s}}}{T_{s}+\bar{T}_{s}}\right\rangle \simeq \frac{m_{3 / 2}}{a \tau_{s}^{(0)}} .
$$

16. Table 2 should be corrected as

\begin{tabular}{|c|c|c|c|c|}
\hline$M_{\mathrm{Pl}}$ & $m_{\tau_{s}}$ & $m_{\rho_{s}}$ & $m_{\mathrm{KK}}$ & $m_{3 / 2}$ \\
\hline 1 & $\mathcal{O}\left(\frac{\ln L_{\text {phys }}}{L_{\text {phys }}}\right)$ & $\mathcal{O}\left(\frac{\ln L_{\text {phys }}}{L_{\text {phys }}}\right)$ & $\mathcal{O}\left(\frac{1}{L_{\text {phys }}}\right)$ & $\mathcal{O}\left(\frac{1}{L_{\text {phys }}}\right)$ \\
\hline$m_{Q_{a}}$ & $M_{r}$ & $m_{\tau_{b}}$ & $m_{q}$ & $m_{\rho_{b}}$ \\
\hline$\lesssim \mathcal{O}\left(\frac{1}{L_{\text {phys }}}\right)$ & $\mathcal{O}\left(\frac{1}{L_{\text {phys }} \ln L_{\text {phys }}}\right)$ & $\mathcal{O}\left(\frac{1}{L_{\text {phys }}^{2}}\right)$ & $\mathcal{O}\left(\frac{1}{L_{\text {phys }}^{2}}\right)$ & $\simeq 0$ \\
\hline
\end{tabular}

17. The paragraph below eq. (4.37) should be replaced with "The mass scales of this model in the unit of $M_{\mathrm{Pl}}$ are summarized in table 2 . We have assumed that $W_{0}=\mathcal{O}(1)$ there."

18. The last three sentences in section 4 are replaced with "Comparing our spectrum in table 2 with (4.40), we find that the nongeometric moduli $\tau_{s}$ and $\rho_{s}$ are heavier than the KK mass scale $m_{\mathrm{KK}}=\pi /\left\langle\hat{\mathcal{N}}^{1 / 2}\right\rangle$ in contrast to (4.40). Thus the expressions of their masses in our analysis are valid only when $W_{0}<\mathcal{O}\left(1 / a\left\langle\tau_{s}\right\rangle\right)$, although our mechanism that realizes a large extra dimension still works even if $W_{0}=\mathcal{O}(1)$."

19. In eq. (B.3), the definition of $T_{R}$ should be corrected as

$$
T_{R} \equiv \bigoplus_{a}\left(-2 d_{a} \cdot \operatorname{Re} T \otimes \mathbf{1}_{n_{a}}\right),
$$

Open Access. This article is distributed under the terms of the Creative Commons Attribution License (CC-BY 4.0), which permits any use, distribution and reproduction in any medium, provided the original author(s) and source are credited. 